\title{
Evaluation of penile cavernosal artery intima-media thickness in patients with erectile dysfunction. A new parameter in the diagnosis of vascular erectile dysfunction. Our experience on 59 cases
}

\author{
Domenico Prezioso, Fabrizio Iacono, Umberto Russo, Giuseppe Romeo, Antonio Ruffo, \\ Nicola Russo, Ester Illiano \\ Department of Urology, University Federico II of Naples, Italy.
}

\begin{abstract}
Summary Objective: A precise characterization of erectile dysfunction (ED) of vascular origin has not yet been achieved, although cavernous peak systolic velocity (PSV) is generally considered a major parameter. Nevertheless the penile dynamic color Doppler is invasive and linked to several complications. The intima-media thicknesses (IMT) of cavernosal artery would add to the predictive value of vasculogenic ED risk and outcomes. We also hypothesized the existence of a correlation between IMT cavernosal artery and IMT carotid arteries. This study seeks to evaluate these hypotheses with our experience, investigating the predictive accuracy of carotid and cavernosal Doppler ultrasound findings for discriminating patients with vasculogenic ED.

Material and methods: A total of 59 subjects (32 vasculogenic ED patients - group A - and 27 no vasculogenic ED patients - group B) were evaluated in our andrological center from September 2012 to June 2013 and enrolled in the study. All subjects underwent medical history, erectile function domain of the International Index of Erectile Function, physical examination, routine and sex hormone blood tests, and high resolution dynamic color Doppler ultrasound evaluation of carotid and penile districts and valutation of IMT in both districts.

Results: The values of cavernosal artery IMT in group $A$ were higher than in group $B(0,28 \pm 0,06 \mathrm{~mm}$ vs $0,17 \pm$ $0,07 \mathrm{~mm}$ ). Even the values of carotid artery IMT in vasculogenic ED group were higher than in no vasculogenic ED group $(0,74 \pm 0,14 \mathrm{~mm}$ vs $0,59 \pm 0,11 \mathrm{~mm})$. The cavernosal IMT showed a moderate $(r=0.61)$ positive linear correlation $(p<0.001)$ with the carotid artery IMT.

Conclusions: An increased cavernous IMT might predict ED of vascular origin with more accuracy than PSV and could be a sensitive predictor also for systemic atherosclerosis at an earlier phase.
\end{abstract}

KEY WORDS: Intima media thickness; Vascular erectile dysfunction; Endothelial dysfunction.

Submitted 19 August 2013; Accepted 5 October 2013

\section{INTRODUCTION}

Erectile dysfunction (ED) is a pervasive disorder that afflicts as many as 30 million men in the United States (1), with an estimated 100 million men affected worldwide $(2,3)$.The risk of ED is related to many factors, including age, smoking, diabetes, heart disease, depression, and hypertension $(4,5)$. Vascular disease is by far the most common cause of ED (6) Formerly dismissed as a psychological condition, ED has now assumed center stage as a readily treatable disorder and a powerful risk-marker for cardiovascular disease (CVD) $(6,7)$. Infact because CVD and ED share etiologies as well as pathophysiology (endothelial dysfunction) and because of evidence that degree of ED correlates with severity of CVD, it has been postulated that ED is a sentinel symptom in patients with occult CVD (8). Endothelial dysfunction is intimately linked to atherogenesis and increased CVD risk (9).

Dysfunction arises following alteration in the release of several vasoactive factors, mainly nitric oxide (NO), from endothelial cells $(9,10)$. Endothelial dysfunction due to an abnormality in the release and/or action of NO is characterized by vasoconstriction, coagulation, increased leucocyte adhesion and stimulation of smooth muscle (SM) cell growth, and is, therefore, central to atherogenesis (9). Several traditional cardiovascular risk factors, such as aging, smoking, hypertension, dyslipidemia and diabetes, and some less traditional risk factors, including inflammation, hypoxia, oxidative stress and homocysteinemia, are related to endothelial dysfunction $(11,12)$. Therefore given that endothelial dysfunction predates atherosclerosis development, this possibility is consistent with the so-called 'artery size' hypothesis (13). This theory posits that atherogenesis is likely to present earlier with clinical symptoms in arteries of a smaller diameter, such as in the penis, than in larger sized arteries, such as in the coronary circulation (14). In as much as the vascular disease is the most common cause of ED, after an intracavernosal injection of a vasodilatory agent, color Doppler sonography is performed to evaluate cavernosal arteries and dorsal vessels, and to demonstrate both arterial insufficiency (primary diagnostic criteria for arterial 
insufficiency include a peak systolic velocity (PSV) of less than $25 \mathrm{~cm} / \mathrm{sec}$ and waveform dampening) (15) and venous incompetence (most investigators used to diagnose venous leakage when arterial end-diastolic velocity is greater than $5 \mathrm{~cm} / \mathrm{sec}$ ) (15). That makes it a valuable tool in the diagnostic evaluation of ED (15). Color Doppler ultrasonography is a valuable, informative and minimally invasive tool in the diagnosis of ED (16). Color Doppler imaging offers several advantages over duplex imaging, including rapid localization of the cavernosal artery and accurate angle correction; depiction of cavernosal artery and dorsal vein flow progression; and demonstration of venous flow and arterial variants (15). However, due to the common pathogenesis that characterizes ED and CVD, the classical color Doppler could be complemented with a new method in use in the study of atherosclerosis of the carotid arteries, the intima-media thickness (IMT). Carotid-wall IMT infact is a surrogate measure of atherosclerosis (17) associated with cardiovascular risk factors (18) and with cardiovascular outcomes (19-22). It is the distance from the lumen-intima interface to the media-adventitia interface of the artery wall, as measured on noninvasively acquired ultrasonographic images of the carotid arteries (20). The IMT is increasingly used as a surrogate end point of vascular outcomes in clinical trials aimed at determining the success of interventions that lower risk factors for atherosclerosis and associated diseases (stroke, myocardial infarction and peripheral artery diseases, like disease of cavernosal artery). We hypothesized that the IMT of cavernosal artery would add to the predictive value of vasculogenic ED risk and outcomes. We also hypothesized the existence of a correlation between IMT cavernosal artery and IMT carotid arteries. This study seeks to evaluate these hypotheses with our experience, investigating the predictive accuracy of carotid and cavernosal Doppler ultrasound (CDU) findings for discriminating patients with vasculogenic ED.

\section{Material AND METHOdS}

The study design consisted of a observational trial conduced from September 2012 to June 2013. The study was conducted according to the Helsinki Declaration. Written informed consent was obtained from all patients. We enrolled 59 patients, mean age was 55,3 $\pm 3,7$ years. Inclusion criteria were: over 51,6 years of age, male patients with stable marital relations and affected by ED. Exclusion criteria were: International Index of Erectile Function (IIEF) score $\geq 26$, alcoholism, smoking, hypertension, CVD, neurogenic syndrome (multiple sclerosis, multiple atrophy, Parkinson's disease, tumors, stroke, disk disease, spinal cord disorders, polyneuropathy, uraemia), Peyronie's disease, penile fracture, congenital curvature of penis, micropenis, hypospadias, epispadias, hyperprolattinemia, hyper- and hypothyroidism, Cushing's disease, drug assumption (PDE5 inhibitors, intracavernous administration of vasoactive drugs, antihypertensives, antidepressants, antipsychotics, antiandrogens, antihistamines, heroin, cocaine and methadone), radiotherapy (pelvis or retroperitoneum) and lower pelvic surgery (oncological pelvic surgery, lower urinary and genital tract surgery). We enrolled 59 patients presenting at the Andrology Department of our Clinic. At visit patients were evaluated by means of a detailed medical and sexual history. A general (including assessment of Body Mass Index BMI - and blood pressure) and urological objective examination was carried out to identify the presence of any diseases that could interfere with erectile function such as Peyronie's disease, cancer of the penis, hypospadias, epispadias, signs and symptoms suggestive of hypogonadism (small testes, alterations in secondary sexual characteristics, decreased libido), neurological disorders. In all patients in whom an autonomic neuropathy was suspected, especially in patients with diabetes, it was assessed by the evocation of the bulbo-cavernous reflex. Each patient finally performed a rectal examination to search for a possible benign prostatic hypertrophy (BPH) to be associated with the presence of lower urinary yract symptoms (LUTS). In each patient lipid and metabolic levels (triglycerides, total cholesterol, HDL cholesterol, fasting blood glucose), hormone levels (testosterone ( $\mathrm{T}$ ), dihydrotestosterone (DHT), luteinizing hormone (LH), follicle-stimulating hormone (FSH), estradiol, prolactin), clinical examination were evaluated. We asked all patients to complete the International Index of erectile function (IIEF) questionnaire: the IIEF domain was calculated and ED grading was so determined: absence of ED (EF score 26 to 30), mild ED (EF score 17 to 25), moderate ED (EF score 11 to 16) and severe ED (EF score < 10) (23). All patients were evaluated with gray scale ultrasound and color Doppler ultrasound just before injection and 1, 5, 15, 20 minutes after injection and the images were recorded. A $7.5 \mathrm{mHz}$ linear transducer with a mechanical standoff wedge to produce a favorable insonating angle throughout the entire field of view was used for the Doppler ultrasound examinations. The degree of erection was classified into flask erection, tumescence, full erection, rigid erection and detumescence phases by an urologist. Spectrum pattern, peak systolic velocity (PSV), end diastolic velocity (EDV) values and compliance were measured with conventional penile Doppler ultrasound 5 minutes after pharmacological stimulation for each subject. According to the reference levels given in the recommendations of the European Association of Urology (EAU) guidelines, a positive test is a rigid erectile response (unable to bend the penis) that appears within 10 min after the intracavernous injection and lasts for $30 \mathrm{~min}$, and a peak systolic blood flow higher than $30 \mathrm{~cm} / \mathrm{s}$ and a resistance index higher than 0.8 are generally considered normal in a duplex ultrasound of penile arteries (24).

Patients were divided in 2 groups: vasculogenic ED (group A) and no vasculogenic ED (group B) according to color Doppler image findings. The group A was composed of 32 patients, while the group B was composed of 27 patients. IMT values of common carotid artery and of cavernosal artery were calculated in all patients. Intimamedia interface lines were manually traced as continuous lines by a certified reader, and IMT values were calculated (25). The mean IMT of the common carotid artery was measured over a segment of the common carotid artery that was $1 \mathrm{~cm}$ long, located approximately $0.5 \mathrm{~cm}$ below the carotid-artery bulb, and considered not to contain any plaque (i.e., not to have any perceivable protrusion of the 
Table 1.

Characteristics of patients: Vasculogenic ED (group A) and No vasculogenic ED (group B).

\begin{tabular}{|l|c|c|c}
\hline & $\begin{array}{c}\text { Vasculogenic ED } \\
(\mathbf{n}=\mathbf{3 2})\end{array}$ & $\begin{array}{c}\text { No vasculogenic ED } \\
\text { (n = 27) }\end{array}$ & P \\
\hline Age mean (SD) & $53,2 \pm 8,2$ & $49,6 \pm 7,6$ & NS \\
\hline IIEF-5 mean (SD) & $10,8 \pm 3,2$ & $17,4 \pm 4,1$ & $\mathrm{p}<0,001$ \\
\hline BMl mean (SD) & $28,1 \pm 3,1$ & $26,5 \pm 4,5$ & $\mathrm{NS}$ \\
\hline Systolic blood pressure (mmHg) mean (SD) & $143,2 \pm 7,5$ & $134,4 \pm 9,2$ & $\mathrm{p}<0,001$ \\
\hline Diastolic blood pressure (mmHg) mean (SD) & $92,3 \pm 5,1$ & $86,9 \pm 6,1$ & $\mathrm{p}<0,001$ \\
\hline Glycemia (mg/dl) mean (SD) & $114,7 \pm 20,3$ & $103,4 \pm 21,2$ & $\mathrm{p}=0,021$ \\
\hline Total cholesterol (mg/dl) mean (SD) & $193,3 \pm 42,0$ & $176,0 \pm 24,8$ & $\mathrm{NS}$ \\
\hline Cholesterol HDL (mg/dl) mean (SD) & $43,9 \pm 10,2$ & $49,3 \pm 8,4$ & $\mathrm{p}=0,016$ \\
\hline Triglycerides (mg/dl) mean (SD) & $175,3 \pm 30,6$ & $160,2 \pm 17,0$ & $\mathrm{p}=0,013$ \\
\hline
\end{tabular}

BMI: Body mass index; ED: Erectile dysfunction; HDL: High-density lipoprotein; IIEF-5: International Index of erectile function.

artery wall into the lumen) (26). In each individual patient, the measurement was made bilaterally while for the statistical study was considered the media of the two values obtained. IMT of cavernosal artery was measured in the proximal artery, choosing the straight portion that offered the best visualization. Also in this case the measurement was bilateral and the average of the values obtained was used for the study. The measurement was made by the same skilled operator for each patient, using a scanner Philips iU22 xmatrix ultrasound system and a probe L12-5 $50 \mathrm{~mm}$ Broadband Linear Array Transducer with a frequency range from 12 to $5 \mathrm{MHz}$. The results of both groups were compared by Student t test $(p<0.05)$.

\section{Results}

At initial evalutation in the group A the IIEF total score was $10.8 \pm 3.2$, moderate ED, while in the second group the IIEF total score was $17.4 \pm 4.1$, mild ED, $(p<0.001)$. This result was expected and is obviously in agreement with the belief that hemodynamic alterations of the penile vasculature are the factors that can mostly impair erectile function.In the group A the diastolic blood pressure was $92.3 \pm 5.1 \mathrm{mmHg}$ versus $86,9 \pm 6,1$ in group $\mathrm{B}$, while the systolic blood pressure was $143.2 \pm 7.5 \mathrm{mmHg}$ and 134,4 $\pm 9,2$ respectively $(p<0.001)$. Furthermore in the group A glucose $(p=0.021)$ and triglycerides $(p=0.013)$ levels were higher than those in group $\mathrm{B}$, while cholesterol levels were lower. $(p=0.016)$. Infact the glucose levels in group A were $114,7 \pm 20,3 \mathrm{mg} / \mathrm{dl}$ versus $103,4 \pm 21,2$ $\mathrm{mg} / \mathrm{dl}$ in group B. Triglycerides levels were $175,3 \pm 30,6$ $\mathrm{mg} / \mathrm{dl}$ in vasculogenic ED group , and 160,2 $\pm 17,0 \mathrm{mg} / \mathrm{dl}$ in no vasculogenic group. Different trend showed the cholesterol levels infact they were higher in group B $(43,9 \pm$ $10,2 \mathrm{mg} / \mathrm{dl}$ - group A - vs 49,3 \pm 8,4 mg/dl - group B). There were not statistically significant differences between the groups in BMI values $(28.1 \pm 3.1$ vs $26.5 \pm 4.5)$.

Table 1 shows the characteristics of the two groups. These findings supported the association of ED with CVD, atherosclerosis and cardiovascular risk. Table 2 shows the findigs of carotid and cavernosal artery IMT. The values of cavernosal artery IMT in group A were higher than in group $\mathrm{B}(0,28 \pm 0,06 \mathrm{~mm}$ vs $0,17 \pm 0,07 \mathrm{~mm})$. This suggested that hemodynamic functional alterations evaluated in penile color Doppler image were correlated with morphological alterations of cavernosal artery evaluated by ultrasound. Even the values of carotid artery IMT in vasculogenic ED group were higher than in no vasculogenic ED group $(0,74 \pm 0,14 \mathrm{~mm}$ vs $0,59 \pm 0,11 \mathrm{~mm})$.

This result is in complete agreement with the association between carotid atherosclerosis and polydistrectual atherosclerosis like cavernosal atherosclerosis. Lastly we wanted to analyze the possibility of a correlation between the values of cavernous IMT and carotid IMT in our study population. As graphically represented in Figure 1, the cavernosal IMT showed a moderate $(r=0.61)$ positive linear correlation $(p<0.001)$ with the carotid artery IMT. The explanation of these results was that the same risk factors and pathogenesis of vascular injury caused simultaneously endothelial damage in different distrects.

Table 2

Intima-media thickness (IMT) values of common carotid artery and of cavernosal artery.

\begin{tabular}{|c|c|c|c|}
\hline & $\begin{array}{l}\text { Vasculogenic ED } \\
(n=32)\end{array}$ & $\begin{array}{c}\text { No vasculogenic ED } \\
(n=27)\end{array}$ & $\mathbf{P}$ \\
\hline IMT cavernosal artery mean (SD) & $0,28 \pm 0,06$ & $0,17 \pm 0,07$ & $p<0,001$ \\
\hline IMT carotid artery mean (SD) & $0,74 \pm 0,14$ & $0,59 \pm 0,11$ & $p<0,001$ \\
\hline
\end{tabular}


Figure 1.

Correlation between carotid artery IMT and cavernosal artery IMT.

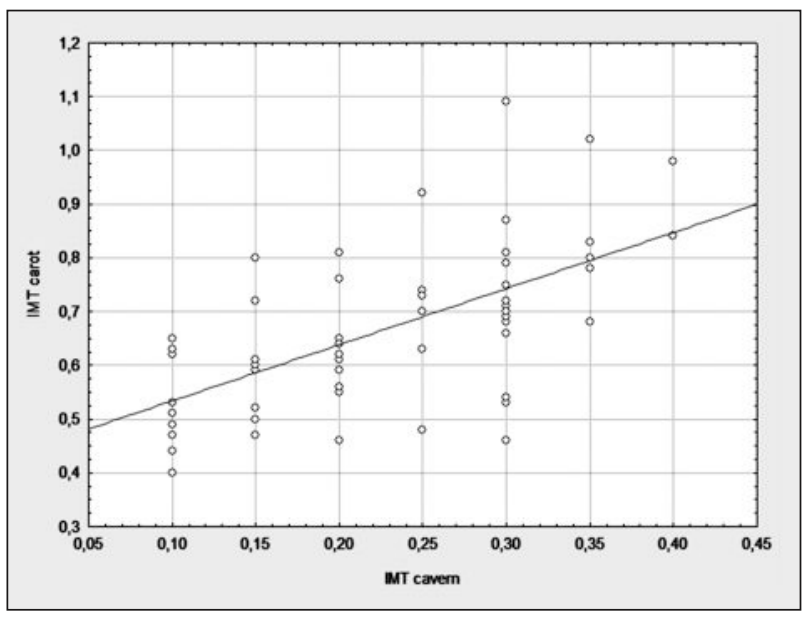

IMT: Intima-media thickness.

\section{Discussion}

The association between ED and CVD has previously been recognized (27). Patients with CVD frequently describe preexisting ED (28). These patients must be clearly distinguished from those who have neither CVD nor cardiovascular risk factors and have a defect in the generating NO-3-5-cyclic guanosine monophosphate peripheral vascular system in SM that is independent of other systemic vascular diseases (29). It has been suggested, but never demonstrated, that early treatment of coronary heart disease risk factors may reduce the later risk of ED (30). Furthermore, it has been hypothesized that ED is a harbinger of CVD $(31,32)$. In a study of men with diabetes with vs without ED, ED was the most efficient predictor of coronary artery disease (33). A largescale study of 25650 men found a $75 \%$ increased risk of peripheral vascular disease in men with preexisting ED (34). Ian M. Thompson demonstrated the substantial association between incident as well as prevalent ED and subsequent CVD, including angina, myocardial infarction, stroke, and transient ischemic attack (35). Several epidemiological studies in selected patient populations have clearly shown that the major cardiovascular risk factors - aging, smoking, diabetes, hyperlipidemia and hypertension - have raised prevalence in individuals with $\operatorname{ED}(36,37)$. The prevalence of $\mathrm{ED}$ is also directly related to the number of cardiovascular risk factors present, being highest in individuals with more than three. Patients with coronary artery disease have a high frequency of $\operatorname{ED}(38,39)$ which correlates with the number of stenotic and calcified arteries and predates symptomatic disease (40). Notably, a patient with vasculogenic ED is likely to have one coronary artery with a 50\% stenosis $(38,39)$. Cardiometabolic risk in abdominally obese subjects is now well-defined as the metabolic syndrome (41). ED prevalence increases with the number of components of the metabolic syndrome, being as high as $40 \%$ in individuals with four components, and is especially prevalent in those with diabetes (42). In individuals with the metabolic syndrome, ED has a linear rela- tionship with evidence of endothelial dysfunction (43). A plausible theoretical link between erectile and endothelial dysfunction posits that cardiovascular risk factors could induce ED by impairing NO release from endothelial cells following neuronal activation and initiation of a penile erection. A residual, important question, however, is whether ED reflects endothelial dysfunction independent of traditional cardiovascular risk factors (43). Carotid-artery IMT, measured noninvasively with the use of carotid-artery ultrasonography, is an independent predictor of new cardiovascular events in persons without a history of CVD (43). A review of eight epidemiologic studies showed that the IMT of the common carotid artery by itself (in all eight studies) or combined with the IMT of the internal carotid artery and presented as a score (in one of the eight studies) had independent predictive power with respect to cardiovascular events (44). The presence of plaque (defined as an internal-carotid artery IMT $\geq 1.9 \mathrm{~mm}$ ) has been shown to be associated with increased event rates (45).

Gokkaya investigated the predictive accuracy of carotid and CDU findings for discriminating patients with vasculogenic erectile dysfunction (EDV). Of total 50 patients, $29(58 \%)$ were included in vasculogenic ED group and 21 (42\%) in non-vasculogenic ED group according to $\mathrm{P}-\mathrm{CDU}$ findings. There was a significant difference between groups for cavernosal IMT $(\mathrm{P}=0.012)$ but not for carotid IMT $(P=0.601)$. When patients were reclassified according to carotid IMT values (IMT of the first group $<0.9 \mathrm{~mm}$ and the second $>=0.9 \mathrm{~mm}$ ), carotid PSV and EDV values were different $(P=0.033$ and 0.018 , respectively). Cavernosal PSV and EDV displayed no difference ( $\mathrm{P}=0.816$ and 0.123 ) while cavernosal IMT and percent change of cavernosal caliper were significantly different $(P=0.014$ and 0.018$)$ (46). Caretta performed a high magnification ultrasonographic study in order to compare functional and morphological parameters of the cavernous artery to PSV and their relation with penile and systemic atherosclerosis (47). A total of 109 subjects (84 ED patients and 25 controls) were evaluated. Cavernous parameters were significantly different between ED and controls. Multivariate model showed that IMT was the only predicting parameter for ED of vascular origin. Cavernous IMT showed a strong direct correlation with carotid and femoral IMT. ED patients with two or more cardiovascular risk factors had a significantly higher cavernous IMT (47). Ucar investigated the relationship between penile color Doppler sonography (CDS) findings and sonographic endothelial parameters in patients with ED, including IMT of common carotid arteries (CCA) and flow-mediated dilatation (FMD) of brachial artery (48). Fifty-six ED patients were included in the study. IMT values were higher in arterial/combined insufficiency group when compared to cavernous veno-occlusive disease but the difference was not statistically significant. The combined use of IMT and FMD established the diagnosis of vasculogenic ED with $100 \%$ sensitivity and $59.2 \%$ specificity. The positive predictive value was $72 \%$, negative predictive value $100 \%$ and accuracy $80 \%$. The combined use of brachial artery FMD and carotid arteries IMT measurements may be suggested as an alternative method to evaluate vasculogenic ED (48). Vlachopoulos evaluated 
arterial structural and functional characteristics and measured systemic endothelial/inflammatory markers in 52 hypertensive men with vasculogenic ED and in 34 hypertensive men with normal erectile function, matched for age, blood pressure, risk factors and treatment (49). Hypertensive patients with ED had higher common carotid IMT $(0.95 \pm .19$ vs. $0.83 \pm 0.18 \mathrm{~mm}, \mathrm{P}=0.003)$ and carotid-femoral pulse-wave velocity $(8.89 \pm 1.38$ vs. $8.11 \pm 1.10 \mathrm{~m} / \mathrm{s}, \mathrm{P}=0.007$ ), lower flow-mediated dilation of the brachial artery (absolute values of $2.96 \pm 1.64$ vs. $4.07 \pm 1.68 \%, \mathrm{P}=0.003)$. In hypertensive men, the presence but not the severity of vasculogenic ED is associated with subclinical atherosclerosis, impairment of arterial function and systemic endothelial and inflammatory activation (49).

\section{Conclusion}

The increase of IMT is an expression of morphological and structural alterations of the vessel wall due to atherosclerotic phenomena. Both the carotid and cavernous IMT proved significantly higher in patients suffering from ED vasculogenic, in association with the classical risk factors for CVD.

In particular, the evaluation of IMT cavernous arteries could be done, together with the color-Doppler evaluation of PSV, by EDV, a new sonographic parameter useful in identifying those cases of vasculogenic ED in a more precise way than what can not be done with the alone assessment of PSV.

The penile color Doppler with intracavernous injection of vasoactive substances is a diagnostic method for a long time widely used in the evaluation of patients with ED. However, it is invasive and linked to several complications such as bruising, hematoma and penile priapism, not to mention that this method is considered embarrassing by most of the patients. In addition, the anxiety that comes from intracavernous injection can induce an abnormal response in the patient who may have adrenergic inhibitory effect, in fact decreasing the sensitivity of this test.

Further studies should be aimed at understanding if the assessment of cavernous IMT is a reliable screening test for those subjects with multiple cardiovascular risk factors at risk of vasculogenic ED, or even if it can completely replace the more invasive and less tolerated colorDoppler investigation with intracavernous injection in the instrumental assessment of erectile function.

\section{REFERENCES}

1. Lewis RW. Epidemiology of erectile dysfunction. Urol Clin North Am. 2001; 28:209-216.

2. NIH Consensus Development Panel on Impotence. Impotence. JAMA. 1993; 270:83-90.

3. Zusman RM, Morales A, Glasser DB, Osterloh IH. Overall cardiovascular profile of sildenafil citrate. Am J Cardiol. 1999; 83:35C-44C.

4. Johannes CB, Araujo AB, Feldman HA, Derby CA, et al. Incidence of erectile dysfunction in men 40 to 69 years old: longitudinal results from the Massachusetts Male Aging Study. J Urol. 2000; 163:460-463.
5. Moinpour CM, Lovato LC, Thompson IM Jr, et al. Profile of men randomized to the prostate cancer prevention trial: baseline healthrelated quality of life, urinary and sexual functioning, and health behaviors. J Clin Oncol. 2000; 18:1942-1953.

6. Shabsigh R, Anastasiadis AG .Erectile dysfunction. Annu Rev Med. 2003; 54:153-168.

7. Montorsi $P$, et al. Common grounds for erectile dysfunction and coronary artery disease. Curr Opinion Urol 2004; 14:361-365.

8. Le NA. Inflammation, oxidative stress, and atherosclerosis. Curr Opin Lipidol. 2004; 15:227-229.

9. Bonetti PO, et al. Endothelial dysfunction: a marker of atherosclerotic risk. Arterioscler Thromb Vasc Biol 2003; 23:168-1757.

10. Cooke JP, Dzau VJ. Nitric oxide synthase: role in the genesis of vascular disease. Annu Rev Med 1997; 48:489-509.

11. Celermajer DS, et al. Endothelium-dependent dilation in the systemic arteries of asymptomatic subjects relates to coronary risk factors and their interaction. J Am Coll Cardiol. 1994; 24:14681474.

12. Brunner $H$, et al. Endothelial function and dysfunction. Part II: Association with cardiovascular risk factors and diseases. A statement by the Working Group on Endothelins and Endothelial Factors of the European.Society of Hypertension. J Hypertens. 2005; 23:233-246.

13. Gerald F Watts, at al. The erectile-endothelial dysfunction nexus: new opportunities for cardiovascular risk prevention. Nature clinical practice cardiovascular medicine 2007; 4:263-272.

14. Montorsi $P$, et al. The artery size hypothesis: a macrovascular link between erectile dysfunction and coronary artery disease. Am J Cardiol, 2005; 96:19-23.

15. Fitzgerald SW, at al. Color Doppler sonography in the evaluation of erectile dysfunction. Radiographics. 1992; 12:3-17.

16. Golubunski Aj, Sikorski A. Usefulness of powerDoppler ultrasonography in evaluating erectile dysfunction. BJU Int 2002; 89:779-782.

17. Hodis HN, Mack WJ, LaBree L, et al. Reduction in carotid arterial wall thickness using lovastatin and dietary therapy: a randomized controlled clinical trial. Ann Intern Med. 1996; 124:548-56.

18. O'Leary DH, Polak JF, Kronmal RA, et al. Thickening of the carotid wall: a marker for atherosclerosis in the elderly? Stroke 1996; 27:224-31.

19. O'Leary DH, Polak JF, Kronmal RA, et al. Carotid-artery intima and media thickness as a risk factor for myocardial infarction and stroke in older adults. N Engl J Med. 1999; 340:14-22.

20. Chambless LE, Heiss G, Folsom AR, et al. Association of coronary heart disease incidence with carotid arterial wall thickness and major risk factors: the Atherosclerosis Risk in Communities (ARIC) Study, 1987-1993. Am J Epidemiol. 1997; 146:483-94.

21. Bots ML, Hoes AW, Koudstaal PJ, et al. Common carotid intimamedia thickness and risk of stroke and myocardial infarction: the Rotterdam Study. Circulation 1997; 96:1432-7.

22. Chambless LE, Folsom AR, Clegg LX, et al. Carotid wall thickness is predictive of incident clinical stroke: the Atherosclerosis Risk in Communities (ARIC) study. Am J Epidemiol. 2000; 151:478-87.

23. Cappelleri JC, Rosen RC, Smith MD, et al. Diagnostic evaluation of the erectile function domain of the International Index of Erectile Function. Urology. 1999; 54:346-351.

24. EAU guidelines 2013. 
25. Polak JF, Pencina MJ, Meisner A, et al. Associations of carotid artery intimamedia thickness (IMT) with risk factors and prevalent cardiovascular disease: comparison of mean common carotid artery IMT with maximum internal carotid artery IMT. J Ultrasound Med. 2010; 29:1759-68.

26. Polak JF, O'Leary DH, Kronmal RA, et al. Sonographic evaluation of carotid artery atherosclerosis in the elderly: relationship of disease severity to stroke and transient ischemic attack. Radiology. 1993; 188:363-70.

27. Bai Q, Xu QQ, Jiang H, et al. Prevalence and risk factors of erectile dysfunction in 3 cities of China: a community-based study. Asian J Androl. 2004; 6:343-348.

28. Montorsi F, Briganti A, Salonia A, et al. Erectile dysfunction prevalence, time of onset and association with risk factors in 300 consecutive patients with acute chest pain and -angiographically documented coronary artery disease. Eur Urol. 2003; 44:360-364.

29. DeBusk R. Sexual activity in patients with angina.JAMA. 2003; 290:3129-3133

30. Fung MM, Bettencourt R, Barrett-Connor E. Heart disease risk factors predict erectile dysfunction 25 years later: the Rancho Bernardo study. J Am Coll Cardiol. 2004; 43:1405-1411.

31. Speel TG, van Langen H, Meuleman EJ. The risk of coronary heart disease in men with erectile dysfunction. Eur Urol. 2003; 44:366-370.

32. Liu PY, Death AK, Handelsman DJ. Androgens and cardiovascular disease. Endocr Rev. 2003; 24:313-340.

33. Gazzaruso C, Giordanetti S, De Amici E, et al. Relationship between erectile dysfunction and silent myocardial ischemia in apparently uncomplicated type 2 diabetic patients. Circulation. 2004; 110:22-26.

34. Blumentals WA, Gomez-Caminero A, Joo S, Vannappagari V. Is erectile dysfunction predictive of peripheral vascular disease? Aging Male. 2003; 6:217-221.

35. Ian M. Thompson, at al. Erectile Dysfunction and Subsequent Cardiovascular Disease. JAMA. 2005; 21:2996-3002.

36. Bortolotti A, et al. The epidemiology of erectile dysfunction and its risk factors. Int J Androl. 1997; 20:323-334.

37. Feldman HA, et al. Erectile dysfunction and coronary risk fac- tors: prospective results from the Massachusetts male aging study. Prev Med. 2000; 30:328-338.

38. Greenstein A, et al. Does severity of ischemic coronary disease correlate with erectile function?Int J Impot Res. 1997; 9:123-126.

39. Kloner RA, et al. Erectile dysfunction in the cardiac patient: how common and should we treat? J Urol 2003; 170 (Suppl):S46-S50.

40. Solomon $H$, et al. Relation of erectile dysfunction to angiographic coronary artery disease. Am J Cardiol. 2003; 91:230-231.

41. Grundy SM, et al. American Heart Association; National Heart, Lung, and Blood Institute Diagnosis and management of the metabolic syndrome: an American Heart Association/National Heart, Lung, and Blood Institute Scientific Statement.Circulation. 2005; 112:2735-2752.

42. Esposito K, et al. High proportions of erectile dysfunction in men with the metabolic syndrome. Diabetes Care. 2005; 28:1201-1203.

43. Giugliano F, et al. Erectile dysfunction associates with endothelial dysfunction and raised proinflammatory cytokine levels in obese men J Endocrinol Invest. 2004; 27:665-669.

44. Lorenz MW, Markus HS, Bots ML, et al. Prediction of clinical cardiovascular events with carotid intimamedia thickness: a systematic review and meta-analysis. Circulation. 2007; 115:459-67.

45. Rundek T, Arif H, Boden-Albala B, et al. Carotid plaque, a subclinical precursor of vascular events: the Northern Manhattan Study. Neurology. 2008; 70:1200-7.

46. Gokkaya CS, Aktas BK, et al. Is there a concordance between carotid and penile cavernosal artery intima-media thickness in patients with erectile dysfunction? International Journal of Impotence Research 2012; 24:44-48.

47. Caretta N, Palego P, at al. Cavernous artery intima-media thickness: a new parameter in the diagnosis of vascular erectile dysfunction. J Sex Med. 2009; 6:1117-26.

48. Ucar $G$, et al. The combined use of brachial artery flow-mediated dilatation and carotid artery intima-media thicknessmeasurements may be a method to determine vasculogenic erectile dysfunction. Int J Impot Res. 2007; 19:577-83.

49. Vlachopoulos C, Aznaouridis, et al. Arterial function and intima-media thickness in hypertensive patients with erectile dysfunction. J Hypertens. 2008; 26:1829-36.

\section{Correspondence}

Domenico Prezioso, MD

dprezioso@libero.it

Fabrizio Iacono, MD

fiacon@tin.it

Umberto Russo, MD

umberto.russo@libero.it

Giuseppe Romeo, MD

giuseppe.romeo85@gmail.com

Antonio Ruffo, MD

antonio.ruffo7@gmail.com

Nicola Ruffo, MD

stoiconk@hotmail.com

Ester Illiano, MD (Corresponding Author)

ester.illiano@inwind.it

Department of Urology - University Federico II of Naples

via Pansini 5 - 80131 Naples, Italy 